\title{
A STUDY ON TECHNOLOGICAL EDUCATION WITH AUGMENTED REALITY
}

${ }^{1}$ Dr. Leelavathi Rajamanickam ${ }^{*},{ }^{2}$ Kate Lam Woon Yee

${ }^{1}$ School of Information Technology, SEGi University

${ }^{2}$ School of Information Technology, SEGi University

*leelavathiraj@segi.edu.my

\begin{abstract}
The objective of having this journal is to analyze about the field of Augmented Reality (AR), the upcoming three-dimensional (3D) virtual objects into real-time three-dimensional (3D) environment in the education environment. Apart from the education application, there are also medical, manufacturing, entertainment and military applications that have been explored. This article describes the features of Augmented Reality system by including types of augmented reality and the benefits from it. Life of the lecturer and student getting bored day by day without any new sort of teaching approaches. Basically, this research paper summarizes the current efforts and address these issues. Hence, discussion about the future direction and areas for Augmented Reality in the field of education. direction and areas for Augmented Reality in the field of education. A new way of learning and teaching can definitely increase the interest of the parties. This research paper provides a starting point for anyone who interested in changing the classic ways of educations into a technological one filled with AR.
\end{abstract}

Keywords: augmented reality; virtual; materials; technologies 


\section{Introduction}

The word "augment" in augmented reality means to add something. With augmented reality (AR), elements like graphics, sounds, and touch feedback are added to our lives. Elements in the virtual world are brought into the real world and are augmented with superimposed computergenerated images to enhance our current perception of reality like the things we see, hear and feel. Unlike virtual reality which uses the entire virtual environment, augmented reality uses the existing environment and has virtual information overlaid on top of it. There are various types of technologies that form augmented reality:

\section{1) Marker Based Augmented Reality}

It is an image recognition technology. The result is produced only when the reader senses a marker using camera or visual marker like QR code.

\section{2) Markerless Augmented Reality}

It is a location-based technology that provides information based on current location using GPS that is embedded in the device.

\section{3) Projection Based Augmented Reality}

It allows human interaction by projecting artificial light onto real environment surface and senses user interaction of that projected light such as touch.

\section{4) Superimposition Based Augmented Reality}

It is an object recognition technology that replaces the original view with a new augmented view of the same object partially of fully.

Today, there are countless reasons to use augmented reality in our lives. It brings convenience and makes a lot of our daily tasks easier. It can basically bring anything virtual to improve our senses of reality.

Most women have to go through multiple sample tests or struggle to find the best makeup product that suits them before buying them. However, most people realize that the products does not really suits them, by the time when they got home. The Shiseido Makeup Mirror is created by the Japanese beauty brand, Shiseido. It gives users a virtual full make-over in seconds and 
allows them to virtually test many different makeup products on eyes, lips and cheeks. The face of the user will be captures by a camera on the device and using the touch screen device, user can choose many different eye, lip colors and blushers. Users can also capture a few still images to be stored and compared. Hence, users can easily find the best cosmetic products for themselves.

Choosing and imagining how furniture will look like in the house is not easy. IKEA discovered one problem that its customer's face, which is the furniture purchased occurs to be the wrong size for the desired location. Therefore, IKEA launched its augmented reality catalogue that offers shoppers a sneak peek of how certain items will look like in their home before purchasing. The app is also able to measure the product size against the room to provide a true-to-life size vision. Different items can be selected, repositioned and viewed from different angles to determine the best product to purchase. Items can be ordered directly using the links integrated into the app.

\section{Literature Review}

A) Observation

Through the method of observation, the current educational system have its flaw from the lecturer's point of view. Due to lack of spaces in classrooms, the students have been split into multiple groups and they are to attend different classes at different time and venue. Such cases, leads to the lecturers teaching the exact same syllabus to multiple classes. The lecturers are to repeat the exact same topics and examples, over and over in different classes at different time and places.

B) Life of Lecturer

Lecturer's life is as it is a tough and tiring ones. Teaching is very time consuming and can be very dispiriting [1]. Especially when a lecturer keep repeating the same thing in every class and similar type of questions asked by different students from different classes. The lecturer had to attend multiple classes teaching the same syllabus is indeed time consuming. It would have been better if the students all could fit in one or two classes in total which would save time for the lecturers. Good teachers in any setting encourage their students [2]. Furthermore, by constantly maintaining a good mood, the lecturer will be good toward their students always. They will 
encourage their students to study and learn things in different ways. In order to ensure a better education, the augmented reality implementation in teaching is required.

\section{C) Virtual Reality}

Virtual reality as the word mentioned it is a fake reality or can be defined as a reality which doesn't exist. Virtual reality technically is a three dimensional environment created by the computer. The created environment can be interacted by a person. The person then becomes a part of the virtual world and can control objects or perform actions. Requires a head mounted display apparatus for a virtual reality system [3]. The devices are known as Head Mounted Display (HMD). It is a devices required in order for the information to be displayed near to eye for the user. Optical components such as lenses are used to provide perception to the user that the visual are occurring from a greater distance, in order to avoid eyestrain. In HMDs which uses a single display, the image is typically displayed via optics that split the visual into two similar visual, and moves each visual sight to the respective eyes. With two displays, the HMD can show stereoscopic visuals [3]. The stereoscopic sight attempt to cause depth in the sight by imitate the angular difference between the visual sights viewed by each eye while looking at an object. Furthermore, angular difference is one of the important parameters of the human brain which is used in processing the visuals to cause distance in human vision or depth perception. The importance of virtual reality can be described with the following example. A group of students wearing 3-D glasses made by Nvidia, a graphics processing firm, dissected a virtual cadaver projected on a screen [4].

\section{D) Augmented Reality}

Augmented Reality (AR) involves the integration of virtual content into real environments [5]. Augmented reality is not exciting as a virtual reality, but, it might be a very useful tool in the everyday lives of humans. AR holds this capabilities because it brings the elements of virtual world and form it into the real world. It allows the people to see, hear, and feel in an enhanced way. Comparing to the other reality technologies, AR is in the middle of the virtual and real world. Augmented reality uses the existing natural surroundings and simply covers virtual information on top of it. When both virtual and real world mixed in together, users experience a new improved world where virtual details or information is used as a tool to present help in everyday activities of a typical human. There is about 4 types of augmented reality. Which is marker based 
augmented reality, markerless augmented reality, projection based augmented reality, and superimposition based augmented reality.

E) Projection based augmented reality

Projection based augmented reality works by casting artificial light into the real world surroundings. Projection based augmented reality application allows human interaction by casting light into a real world surrounding and then detecting the human interaction of that casted light. Detecting the user's interaction is possible by addressing difference between a known projection and the changed projection caused by user's interaction which is blocking the projected light. Another application of projection based augmented reality which catches the attention is, it utilizes laser plasma technology to cast a three dimensional interactive hologram in to the middle of the air. Key components for augmented realities are sensors, cameras, projection, processing, and reflection [6]. There are few existing projections, which is used for educational purposes of medical science. In medical terms, the surgeons. Head mounted displays have become more accessible and more affordable [7]. Augmented Reality have the potential to expand surgical precision, expand patient safety, and provide physician education.

\section{F) Augmented Reality in Education}

Augmented Reality will definitely transform Education as we knew it one day. The capabilities to project rich media into the real world, in order to view through web enabled devices. By using mobile phones and tablet devices, information can be made available and accessible to students at the time and place of needs. Moreover, potential to cut down excessive cognitive, as well as enabling them to learn in a range of different ways. For example while reading a paragraph from a device or a book, a 3D video will be displayed in their handheld device portraying how the lecturer or instructor explained the solutions to be for a problem. There are plenty of AR applications which changed the ways of learning and training. Augmented reality provides students with "perfectly situated scaffolding" [8]. One of the outmost importance of educational environments is to practice and familiarise social interaction between the students, whom are present at the same area. Multiple users can access a shared area filled with virtual objects, even though remaining in the real world [9]. 
Augmented reality was defined in a more detailed manner where "augmented reality is the interaction of superimposed graphics, audio and other sense enhancements over a real-world environment that is displayed in real-time" [15]. Augmented reality (AR) is specifically considered as a mix of virtual reality and real life; it is also able to produce images that allows the content to mix with the real world. With these features, the virtual world with the real world can be connected by the users but they are still able to distinguish between both virtual and reality [10]. AR has the ability to produce the illusion of reality using different human senses. In the multi-sensory environment, participants can experience the sensation of sight, hearing, smell and even touch. [16].

Simultaneously, augmented reality is also commonly known as a new technology which allows overlaying of virtual images on the real environment. Augmented reality an environment where the additional virtual information is computer-generated and is placed into the user's real world perception. This environment can be shown in the following example. According to the image in Figure 1, we can see a girl having her breakfast sitting by the table and she is in the real environment. Meanwhile, cartoon character that we can see is a virtual object that overlays on the real world view. It creates an impression to make the viewer believes that the cartoon character next to the girl is real.

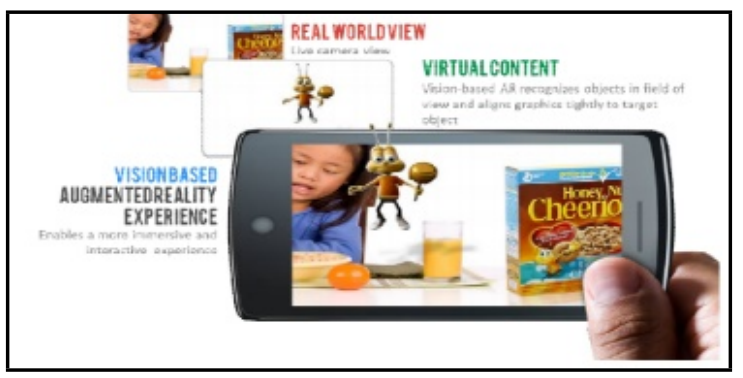

Figure 1 Real World View

The phrase virtual reality (VR) according to [18], is defined as the following collection of technological devices: a computer capable of interactive 3-dimensional (3D) visualization, a head-mounted display (HMDs), and controllers equipped with 1 or more position trackers. Apart from that, the term virtual reality according to [17] is considered as an artificial environment, which is experienced through sensory stimuli, provided by computer when the user's action 
partially determines as to what would happen inside the environment. Virtual reality was actually a version upgraded from augmented reality, when Tom Caudell merged the technology from virtual image to physical reality for aircraft usage back in 1990.

Virtual Reality is commonly defined as using 3D modeling software to create realistic 3D scene of the real world environments to bring and merge the users into this virtual world. It makes tricks on user's brain and make them believe that they are in this virtual world and keeps them from realizing the real world. Virtual reality replaces our real senses such as touch, sight and hearing with the virtual world so that our brains start to believe that the virtual world itself is the real world.

To experience virtual reality, there are four key elements:

i. The virtual world is an imaginary room and it is often but not essentially realized through a medium or display.

ii. Immersion through physical and mental, which means having a sense of "presence" within an environment. This can be done through physical means or a mental state.

- Mental Immersion: a state of being deeply immersed in the virtual environment.

- Physical Immersion: entering into a projected area physically

iii. Sensory feedback: sight, hearing and touch responses.

iv. Interactivity: in a virtual reality experience, users will make movements using their head to change their points of viewing.

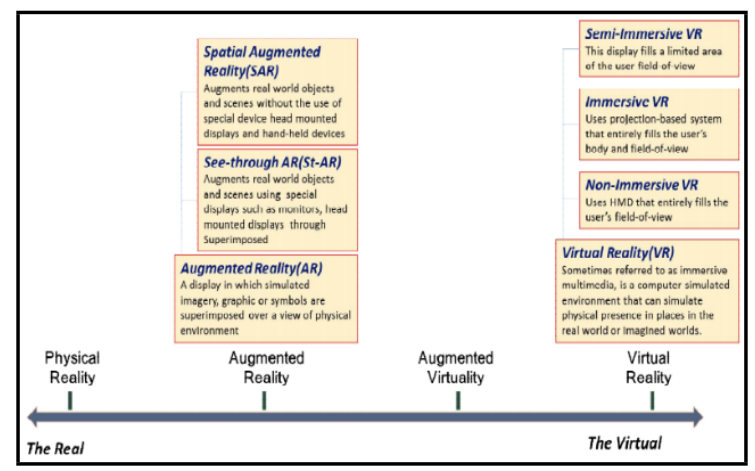

Figure 2 The Real to Virtual world phases 
Both the AR and VR are the dominants of the new technologies and are believed to be the fascination and the world's most influential technology. Virtual reality is able to invert and shift the user's viewpoints or in other words, it can bring people to someplace else. VR blocks out the reality and locates our presence somewhere else in the virtual environment by using closed visors or goggles. The application of virtual reality in films is such as "The Matrix", whereas augmented reality film will be such as "The Terminator". The purpose of VR is to convince the viewers of being in an entirely new reality by using illusion.

Comparatively, augmented reality does not locate the sense of the real world, but it is improved by using computers. Both the VR and AR technologies have one main difference. VR is immersive, which is the display device must block out the real world entirely. AR does not necessarily need headsets and it overlays a virtual object partially on the real world, which maintains the users' connection with the real world.

The appearance of AR or VR could bring benefits to various industries such as entertainment, gaming, medication and education. In conclusion, both of them have one common area of similar, which is they both have the ability shift our perceptions of the real world. Whereas their difference will be the perception of the presence of the real world.

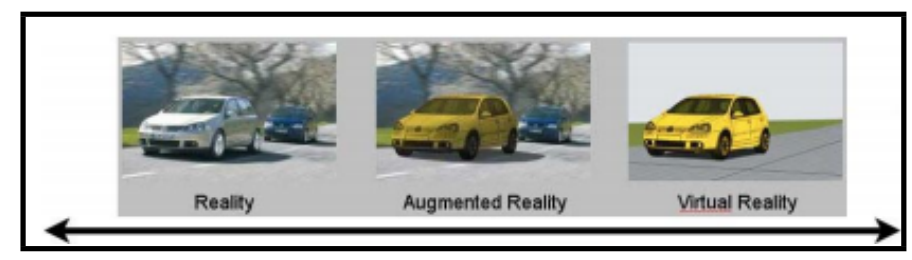

Figure 3 Schema of a Virtuality Continuum

\section{Research Methodology}

\section{A) Materials}

A product which can be used as an object physically in order to attain augmented reality experience. There are various types of Materials which can be used for augmented reality. For example, Head Mount Device (HMD) which helps user to visualize virtual images onto the real physical world. Augmented Reality (AR) which overlaps virtual objects onto real scenes, and it 
has a large platform which provides the students with a new type of materials. Augmented instructions is a fusion of augmented reality and printed learning materials.

\section{B) SmartPhone}

As the dependency on situation, the position sensors in smartphones and tablets are not only augmented reality based but also to meet educational requirement. The dependency of these sensors are very important. Previous researches have measured accuracy of the position sensors (usually face detection) in portable devices. This aggregation includes handheld video or optical radiology displays and handheld projectors. Although such displays are bulky than headmounted displays, they are currently the best solutions for bringing Augmented Reality to the industry because of their low manufacturing cost and ease of use [11]. Augmented Reality tools and techniques for developing interactive materials for mobile-learning.

\section{C) Projective}

The technology analyze the individual's psychodynamic profile by presenting unstructured or obscure materials such as ink, images, and sentence elements, that will trigger an interpretive response. Which

reveals the personality structure because they do not require specialized glasses. They accommodate the user's eyes during focusing, and they can cover large surfaces for a wide field of view.

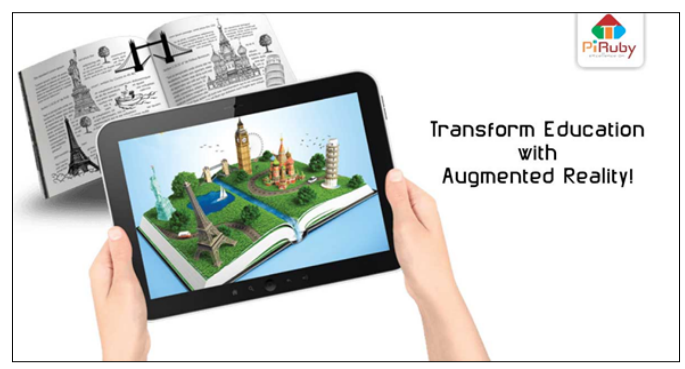

Figure 4 Transform Education with Augmented Reality

However, like the optical transparent displays, mediate fundamental interaction requires other input devices. Thus, the projector needs to be calibrated each time the environment or 
distance on the projection surface changes which is crucial in moving settings. In addition, this type of display is limited to indoor use due to the low brightness and contrast of projected images.

This can be improved by covering the surface with a reflective material. The objects and instruments contained in this material will reflect directly to the light source near the viewer's eye and will therefore not interfere with the projection.

D) Hand-Held

This accumulation includes handheld video or optical perspective monitors and handheld projectors for Augmented Reality. This is a study which shows how hand-held products are used in Augmented Reality. Although this type of monitors are larger than head mounted displays (HMD), It shows that this is the best solution to present an Augmented Reality because of its low production costs, easy to use(user friendly) and it has become a physical property in the industry. For example, handheld video perspective Augmented Reality as a magnifying glass can be based on future consumer's passage such as cell phones that show 3D objects [11]. We believe that an unconstrained, structure-independent Augmented Reality demonstration would be needed to bridge the gap with traditional packing systems. Specific example includes unpredictable use over long time spans, mixed indoor with outdoor, used in a wide-area environment, and large-scale multi-user scenarios. This has motivated us to develop a state of the art AR framework targeting lightweight handheld displays

\section{E) Spatial}

Spatial in augmented reality is a fast rising area that everyone cares about. Any types of digital art work improvement, digital media work improvement and interested in the display technology are the impact of computer graphics. We believe in different rich pallets displayed in technology, mobile and non-mobile, must be considered and appropriate for a given application so that you can choose the most efficient technology. Spatial Augmented Reality Merging Real and Virtual Worlds. The final type of display is statically placed inside the environment and includes screen-based video perspective display, space optical perspective display and projection monitor. These technologies are suitable for large education presentations, exhibitions and limited interaction. The way to create an Augmented Reality is based on a fine traditional screen (PC or TV) display with an Augmented Reality camera fixed cover. This technology is now being applied in the world Sports TV for the environments like swimming pool. The swimming pool and track are very clear and easy to augment [11]. 


\subsection{Methodology}

A method used in a particular research area or activity. Virtual objects can be displayed as real objects before the Augmented Reality system environment.

\section{A) Tracking sensors and approaches}

The system must be able to understand the environment and preferably lead the relative movement of the viewer's Six Degrees of Freedom (6DOF): Three variables (x, y, and z) and three angles (yaw, pitch and roll direction). In addition, most environments in the Augmented Reality system must be ready before it can be used to track 6DOF movements, but not all tracking techniques might work in all environments. So far, the complete direction of the user is still a single complicated problem [11].

B) Modeling Techniques

Creating a 3D model of a large environment is a challenge itself. 3D modelling techniques can be automatic, semi-automatic and a manual technique. Ray tracing algorithms are more suitable for huge visualization. Moreover, data sets are better than traditional z-buffering algorithms. Some have important research questions regarding sculpture of any complex 3D space model. The social group stores and queries these data as spatial database. These databases may also need to be changed since the real environment is often energizing.

\section{C) Human Computer Interaction}

Information technology currently supports the development of human interaction with the virtual environment. Which will continue to evolve in the form of Human Computer Interaction (HCI). The research shows, how an environmental three-dimensional (3D) projection should recognize the human hand as a virtual object and interact with the virtual environment. Humancomputer interaction is the study of the relationship between people and computer technology, and how computers are designed to make humans life easier, more practical, and more intuitive. HCI emphasizes the interaction between people and computer technology. The study shows, the three-dimensional (3D) transformation of digital objects are used in computer graphics to transform the movement of digital objects. It is possible because of, the interactive technologies existing in the virtual environment. Trackers are needed for AR in virtual interaction. The problem in AR is, how to read tags so it can show previously calculated virtual objects. Mainly 
how to read the human geometry model. The result of the geometry is used as a marker, so it interacts with a virtual environment on the AR as an implementation of the HCI model. HumanComputer Interaction (HCI) areas involves on how humans can interact with information and computing systems. This is a rich domain that has led to the development of modern graphical user interfaces.

\section{Discussion}

Augmented Reality or AR could be a part of the future of education. Its implementation now could be difficult and challenging since current technology is still developing motion tracking technology but could definitely be part of educations future. The way of implementing such technology to classrooms should be through use of virtual reality headsets. Headsets like the oculus rift or vive headset which are currently focused for gaming could be used in the classroom [12]. The devices track motion and display the augmented reality through lens in the headset. This would mean that for every student would require a headset in order to view the class or lecture through AR. Sometimes the requirement for AR does not mean that one needs a headset for augmented reality but a hand phone. Similar like playing Pokémon Go with AR features or Youtube's 360 video function, one can simply view an augmented reality through their mobile devices like mobile phones or tablets. This would allow students to project their lecturer's teaching method anywhere like the realization of having your lecturer teach and explain like from the comfort of a student's living room or bed room or even in public such as a library or in a park.

As for its importance, the use of AR could provide a feel that can't be shown or explained by a teacher in a classroom. For some it could show how certain living creatures live in the wild or experience an event such as a natural disaster up close as this allows the student to feel as if he is near or part of the subject as if he was there. This means that the student can learn through viewing without putting themselves into harm's way. AR could also mean less expense on purchasing $3 \mathrm{~d}$ models or paying for excursions. That means that augmented reality could replace physical materials that could wear and tear or even get stolen. The current and possibly future generation can't do well without their technological gadgets but through the use of AR students can learn many forms of experience interactively. Not only that it would appeal to the students but it would retain their attention and maintain their memory for a long time too. Interaction with AR could also lead to a rise in a student's physical and mental dexterity where some forms of AR allow a student to become part of the program [13]. 
The possible use of such AR programs could benefit different education fields. For those learning in the medical field could be exposed to a new reality such as viewing of a live surgery, learning form live $3 \mathrm{~d}$ organ models or identifying symptoms of illnesses, infections and wounds through virtual models. This gives these students a new way of looking at medical topics other that a book. There is also AR tools for those learning engineering. Possibly through the use of $3 \mathrm{~d}$ modelling and physics allows students to model or develop structures for assignments or view other models of past engineering that were used earlier? This allows the engineering students to learn and create types of structures and identify possible weaknesses. For students learning the art of 3D animation could even develop their own 3D animated character or model. The model could be used to create a future game or animated film. Even for primary students the ability to visualize and interact with AR programs means they would have a deeper understanding and fascination to their subjects. Other than the student using the technology of AR, the teacher or lecturer could record a class for those who are unable to attend class that day. The recording could be saved to a cloud storage and then viewed by all students from their mobile devices.

With the future being uncertain, the possibility for enhancing our current education with AR technology could change what we know. This could happen not in schools but other institutions as well. One example could be places like museums, where historical events could be simulated and shown through an augmented reality. Visitors can experience the past as though they were there increasing their interest in our history. Other than re-enactments of our history, while browsing through the artefacts and models a description will appear to inform the user of what he is looking at, the age of the artefact and the history behind the object. Then there would be places like zoos or aquariums where 3D models of animals can be shown to expand the view on animals like instead of seeing it in real life but discovering their internal systems or their locomotion. Instead of reviewing large amounts of notes a student could scan a section and a virtual teacher could explain and help them understand. This will be the same with homework as a student could scan the question and hopefully get assistance through a virtual teacher. Apart from homework or experiences, AR could help those who are deaf or hard of hearing. The system could display flashcards on notes that could explain hard to pronounce words or even display it in a form of sign language [14]. 


\section{Result}

This article describes the features of augmented reality systems, including a detailed discussion of the trade-off between optical and video blending approaches. Registration and sensing errors are two of the biggest problems when it comes to building effective augmented reality systems. So this article sums up the current efforts to address these issues and to discuss the future direction and areas for further study. Moreover, by enhancing these characteristics of reality to provide a clearer foundation, it can be a beneficial to Augmented Reality developers. The significance of understanding augmented reality is still the foundation for ongoing doctoral research. This research is expected to improve the overall performance of both the lecturers and students by implementing the AR in the lecture classes.

\section{Conclusion}

Augmented reality is another step in the age of information. In the days of future, people can soon see the changes in the environment. Changes will be occurred, via smartphones, glasses, car windscreen, and even the windows to show enhanced content in front of people. This will be an amazing way of life for students and lecturers, since it will be more productive and safer. Perhaps in the future, people will see the environment of augmented reality more useful by displaying information based on man's own interests. Nevertheless, students someday will experience AR as a part of their student's life. Students and lecturers will no longer be able to recognize what is real and what is virtual, and the world of education will be the fusion of digital and physical appearance. Therefore, Augmented Reality will soon be an impact for students and lecturer's daily lives through the multi-function devices such as tablets and smartphones. It is a common occurrence for the devices to cost cheaper every year. So Augmented Reality occupies most of the market in only a matter of time.

\section{References}

[1] Cunningham, A. (2002, February 22). Work and Play: A Lecturer's Life in a New University. Retrieved from Sciencemag: http://www.sciencemag.org/careers/2002/02/work-and-play-lecturerslife-new-university

[2] William B.Wood , \& Kimberly B. Tanner. (2012). PMC3292071. Retrieved from ncbi: https://www.ncbi.nlm.nih.gov/pmc/articles/PMC3292071

[3] Wei Chih Chang. (2015). Virtual reality system. 
[4] Singer, N. (2012, January 7). Busiiness day. Retrieved from nytimes:

http://www.nytimes.com/2012/01/08/business/the-human-anatomy-animated-with-3-dtechnology.html

[5] Hoshang Kolivand. (2016). Retrieving Virtual World Light Data to Real Environments. LivePhantom.

[6] Reality technologies. (2016). Augmented Reality. Retrieved from Reality technologies: http://www.realitytechnologies.com/augmented-reality

[7] M.Rankin, T. (2015). Augmented reality in surgery. Technological advances in Surgery, 59-71.

[8] Bower, M. (2014). Augmented Reality in education - cases, places and potentials. Educational Media International, 1-15.

[9] Kaufmann, H. (n.d.). Collaborative Augmented Reality in Education.

[10] McKalin, V. (2015). Augmented Reality vs. Virtual Reality: What are the differences and similarities?. Retrieved from: http://www.techtimes.com/articles/- 5078/20140406/augmentedreality-vs-virtual-realitywhat-are-the-differences-and-similarities.htm

[11] D.W.F. van Krevelen and R. Poelman. (2010). The International Journal of Virtual Reality. A Survey of Augmented Reality Technologies, Application and Limitation, 1-20.

[12] López, Leticia Lafuente (March 24, 2016) Virtual Reality And Augmented Reality In Education, $\mathrm{https}$ //elearningindustry.com/virtual-reality-augmented-reality-education

[13] Marsh, Anna (24 December 2016) 8 Benefits of using augmented reality in education, https://www.onlinecultus.com/8-benefits-using-augmented-reality-education/

[14] Nesloney, Todd (November 4 2013) Augmented Reality Brings New Dimensions to Learning, https://www.edutopia.org/blog/augmented-reality-new-dimensions-learning-drew-minock

[15] Peddie, J. (2013). Augmented Reality still casting about for the right model. Retrieved from: http:/gfxspeak.com/2013/10/29/augmented-realitystill-casting-about-for-the-right-model/

[16] Mattes, S. (2013). Sensorama. Retrieved from: http://smattes.com/article/47/sensorama

[17] Hoyt, C.L. (2015). Immersive Virtual Environment Techonology

[18] Lanier J, Zimmerman TG, Blanchard C. (1986). A hand gesture interface device; Proceedings of the SIGCHI/GI Conference on Human Factors in Computing Systems and Graphics Interface;1987Apr59;Toronto,CA.1987.pp.189-192. 